\title{
Virtual simulation training for fibreoptic intubation
}

\author{
Sylvain Boet, MD · Viren N. Naik, MD • \\ Pierre A. Diemunsch, MD, PhD
}

Received: 27 October 2008/Revised: 29 October 2008/Accepted: 30 October 2008/Published online: 19 December 2008

(C) Canadian Anesthesiologists' Society 2008

To the Editor,

The fibreoptic orotracheal intubation (FOI) technique is considered the gold standard in the management of predicted difficult airways, and every anesthesia practitioner is required to master this complex psychomotor skill. ${ }^{1}$ There are few opportunities for anesthesiology residents to learn this skill in a clinical setting with patients with "true" difficult airways, and learning FOI with patients with normal airways raises ethical considerations. Consequently, anesthesiology residents may be challenged to gain sufficient FOI experience during the course of their postgraduate training. Computer virtual reality simulation may optimize learning opportunities in the clinical setting by providing an ethical and cost-effective modality to develop the basic skills of airway bronchoscopy. ${ }^{2,3}$

As FOI is a complex psychomotor skill, we have observed that it is difficult for novices to control the movements of the bronchoscope accurately while simultaneously thinking about the anatomy of the upper airway. Computer simulation can enhance learning by functioning as a part task trainer. Part task training is defined as the deconstruction of multicomponent tasks into several single-component tasks. When each skill is learned separately,

The Virtual Fiberoptic Intubation (VFI) software was developed by the Institut de Recherche contre les Cancers de l'Appareil Digestif (IRCAD), Strasbourg, France, in partnership with the University of Strasbourg, France, without any financial support. KarlStorz GMBH (Tuttlingen, Germany) distributes the VFI CD free of charge.

S. Boet, MD $(\varangle) \cdot$ V. N. Naik, MD

St Michael's Hospital, University of Toronto, Toronto, Canada

e-mail: sylvainboet@free.fr

P. A. Diemunsch, MD, PhD

Hôpital de Hautepierre, University of Strasbourg,

Strasbourg, France the opportunity for the subsequent integration and overall success of FOI may be enhanced. Compared to whole task training, the single task format facilitates a more rapid development of automatic skills. ${ }^{4}$

We present a computer simulator that focuses on the part task training of understanding altered airway anatomy. The Virtual Fiberoptic Intubation (VFI) software ${ }^{1}$ (Institut de Recherche contre les Cancers de l'Appareil Digestif, France) was developed from reconstructed images of computerized tomography or from magnetic resonance imaging of actual patients varying in age (adults and pediatrics) and pathology (from a cleft palate to post-tracheotomy tracheal stenosis). This simulation enables a trainee to obtain an internal view of the airway that is similar to the view obtained by a bronchoscopy in an actual patient. The $\mathrm{CD}$ provides trainees with six different virtual patients (Fig. 1).

The screen view of the program is divided into three parts. The main window represents the view from the tip of the fibreoptic bronchoscope. The second and third windows of the screen demonstrate three-dimensional reconstruction with several levels of transparency and radiological views. This representation provides an external position for the fibreoptic bronchoscope from which the internal view has been derived. While the virtual bronchoscopy is being performed from either the mouth or the nose, the virtual navigation facilitates a better understanding of the successive changes in direction essential for successful bronchoscopy. This virtual progression helps the trainee to mentally integrate the schema of the correct airway route by simply using his/her computer's "mouse" or keyboard. A key quality of VFI is that it has no pre-calculated routes;

\footnotetext{
${ }^{1}$ Available free of charge from KarlStorz GMBH with a written communication at: www.karlstorz.com.
} 
Fig. 1 On screen view of the Virtual Fiberoptic Intubation (VFI) software. Upper left panel Three-dimensional reconstruction of the airway with different levels of transparency. The arrow represents the position of the tip of the bronchoscope. Lower left panel Radiological views of the airway that can be rotated on three planes. Right panel Virtual reality view from the tip of the bronchoscope
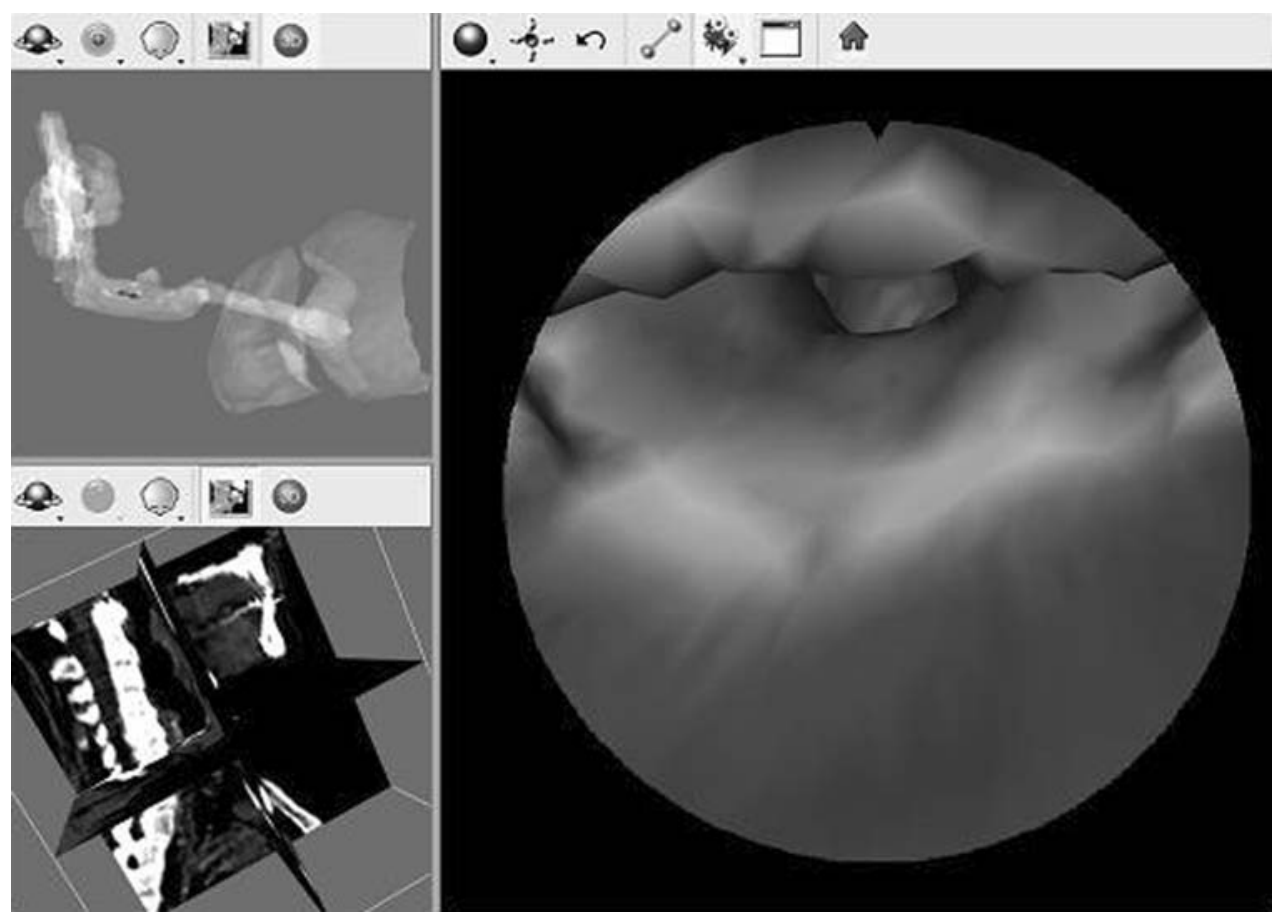

\section{References}

1. American Society of Anesthesiologists Task Force on Management of the Difficult Airway. Practice guidelines for management of the difficult airway: an updated report by the American Society of Anesthesiologists Task Force on Management of the Difficult Airway. Anesthesiology 2003; 98: 1269-77.

2. Rowe $R$, Cohen $R A$. An evaluation of a virtual reality airway simulator. Anesth Analg 2002; 95: 62-6.

3. Goldmann K, Steinfeldt T. Acquisition of basic fiberoptic intubation skills with a virtual reality airway simulator. J Clin Anesth 2006; 18: 173-8.

4. Johnson KB, Syroid ND, Drews FA, et al. Part task and variable priority training in first-year anesthesia resident education: a combined didactic and simulation-based approach to improve management of adverse airway and respiratory events. Anesthesiology 2008; 108: 831-40. 